\title{
Alignment and performance of the Infrared Multi-Object Spectrometer
}

\author{
Joseph A. Connelly ${ }^{* a}$, Raymond G. Ohl ${ }^{\mathbf{a}}$, J. Eric Mentzell ${ }^{\mathrm{a}}$, Timothy J. Madison ${ }^{\mathbf{a}}$ \\ Jason E. Hylan ${ }^{b}$, Ronald G. Mink ${ }^{\mathrm{a}}$, Timo T. Saha ${ }^{\mathrm{a}}$, June L. Tveekrem ${ }^{\mathrm{a}}$, Leroy M. Sparr ${ }^{\mathrm{a}}$ \\ V. John Chambers ${ }^{\mathrm{a}}$, Danette L. Fitzgerald ${ }^{\mathrm{c}}$, Matthew A. Greenhouse ${ }^{\mathrm{a}}$, and John W. MacKenty ${ }^{\mathrm{d}}$ \\ ${ }^{a}$ NASA/Goddard Space Flight Center, Greenbelt, Maryland. \\ ${ }^{\text {b }}$ Swales Aerospace, Beltsville, Maryland.

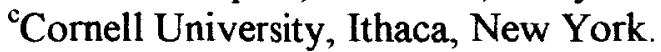 \\ ${ }^{\mathrm{d}}$ Space Telescope Science Institute, Baltimore, Maryland.
}

\begin{abstract}
The Infrared Multi-Object Spectrometer (IRMOS) is a principle investigator class instrument for the Kitt Peak National Observatory 4 and $2.1 \mathrm{~m}$ telescopes. IRMOS is a near-IR $(0.8-2.5 \mu \mathrm{m})$ spectrometer with low- to mid-resolving power $(\mathrm{R}=300-3000)$. IRMOS produces simultaneous spectra of $\sim 100$ objects in its $2.8 \times 2.0$ arc-min field of view ( $4 \mathrm{~m}$ telescope) using a commercial Micro Electro-Mechanical Systems (MEMS) micro-mirror array (MMA) from Texas Instruments. The IRMOS optical design consists of two imaging subsystems. The focal reducer images the focal plane of the telescope onto the MMA field stop, and the spectrograph images the MMA onto the detector. We describe ambient breadboard subsystem alignment and imaging performance of each stage independently, and ambient imaging performance of the fully assembled instrument. Interferometric measurements of subsystem wavefront error serve as a qualitative alignment guide, and are accomplished using a commercial, modified Twyman-Green laser unequal path interferometer. Image testing provides verification of the optomechanical alignment method and a measurement of nearangle scattered light due to mirror small-scale surface error. Image testing is performed at multiple field points. A mercury-argon pencil lamp provides a spectral line at $546.1 \mathrm{~nm}$, a blackbody source provides a line at $1550 \mathrm{~nm}$, and a CCD camera and IR camera are used as detectors. We use commercial optical modeling software to predict the pointspread function and its effect on instrument slit transmission and resolution. Our breadboard and instrument level test results validate this prediction. We conclude with an instrument performance prediction for cryogenic operation and first light in late 2003.
\end{abstract}

Keywords: alignment, infrared, image testing, scatter, MEMS, micro-mirror array, IRMOS

\section{INTRODUCTION}

The Infrared Multi-Object Spectrometer (IRMOS) is a facility instrument for the Kitt Peak National Observatory (KPNO). ${ }^{1}$ It is a collaboration between NASA/Goddard Space Flight Center (GSFC), KPNO, and the Space Telescope Science Institute (STSCI), led by Dr. John W. MacKenty of STSCI. IRMOS will begin testing at the telescope in fall of 2003, and is designed for optimal performance on the 3.8 meter Mayall Telescope at KPNO. IRMOS is a low- to midresolution $(\mathrm{R}=\lambda \Delta \lambda=300-3000)$ spectrometer that will operate in the near-IR $(0.8-2.5 \mu \mathrm{m})$. The instrument (optical bench and mirrors) is machined from aluminum 6061-T651, and operates at a temperature of $80 \mathrm{~K}$. IRMOS uses a commercial micro-electromechanical system (MEMS) multi-mirror array (MMA) from Texas Instruments ${ }^{+}$as a programmable slit mask. The MEMS device will allow IRMOS to produce simultaneous spectra of $\sim 100$ objects in its $2.8 \times 2.0 \mathrm{arcmin}$ field of view. The total weight of the instrument is $\sim 340 \mathrm{~kg}$ and is housed in a cylindrical dewar, $\sim 1$ meter in diameter and $\sim 1.5$ meters high.

IRMOS is the first multi-object spectrometer to use a MEMS device as a programmable slit mask (i.e., field stop). Its primary mission is to serve as an instrument at KPNO. It was also a pathfinder for a possible James Webb Space

\footnotetext{
" Correspondence: Email: Joseph.A.Connelly@nasa.gov, Telephone: 301-286-9527; Fax: 301-286-0204

${ }^{+}$Texas Instruments, Dallas, Texas, tel.: 800-336-5236, http://www.ti.com
} 
Telescope (JWST) MEMS multi-object spectrometer. Science observations at KPNO will be the first to measure the performance of a MEMS device in an astronomy application.

The IRMOS optical design consists of two confocal imaging subsystems that are each comprised of two powered mirrors and one flat, fold mirror (figure 1). ${ }^{2}$ The first imaging system, "stage 1 ," is a focal reducer that relays an off-axis section of the telescope focal plane onto the tilted surface of the MMA. The stage 1 optical system consists of mirrors M1, M2, and F1. Stage 1 changes the f-number from f/15 to $\mathrm{f} / 4.6$. The MMA consists of a $848 \times 600$ array of $16 \times 16 \mu \mathrm{m}$ flat mirrors that all tilt about an axis at $\pm 10^{\circ}$ for "on" and "off" states. The second system, "stage 2," is an imaging spectrograph: the "on" pixels of the MMA are relayed to the surface of a large-format detector. The stage 2 optical system consists of mirrors M3, M4, F2, and a flat grating or flat imaging mirror. Spectra or images are selected by actuating a wheel mechanism. The grating wheel mechanism can select 1 of 13 different flat gratings, or a flat mirror for imaging mode. When the instrument is in imaging mode, near angle scatter and misalignment broaden an imaged point. When in spectrograph mode, these factors decrease spectral purity. It is critical to measure point spread function (PSF) degradation due to mirror surface error. The MMA aiso contributes to PSF degradation, and its contribution must be distinguishable from that due to the mirrors in order to assess its usefulness to JWST. Optical component fabrication and testing are discussed by $\mathrm{Ohl}$ et al. and Chambers et al. ${ }^{3,4}$ System optical prescriptions and tolerances are discussed by Connelly et al. ${ }^{5}$

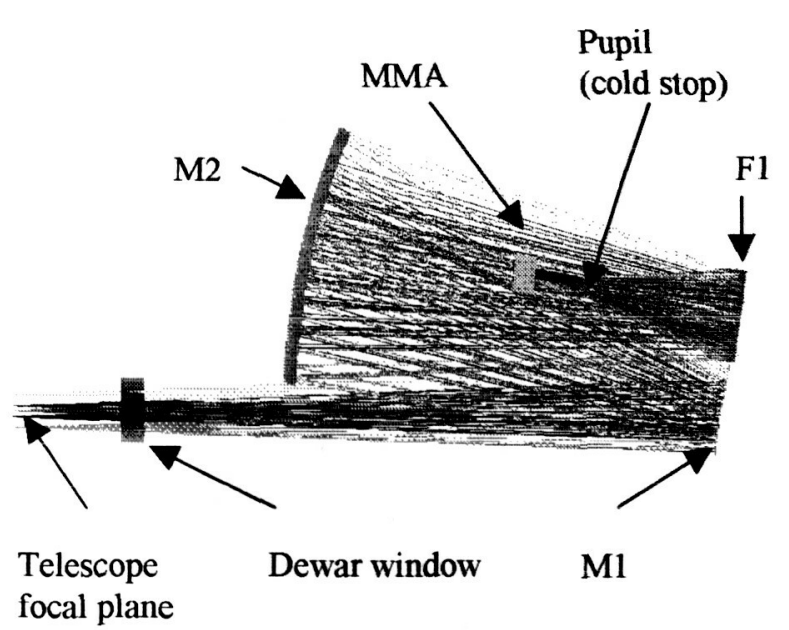

(a)

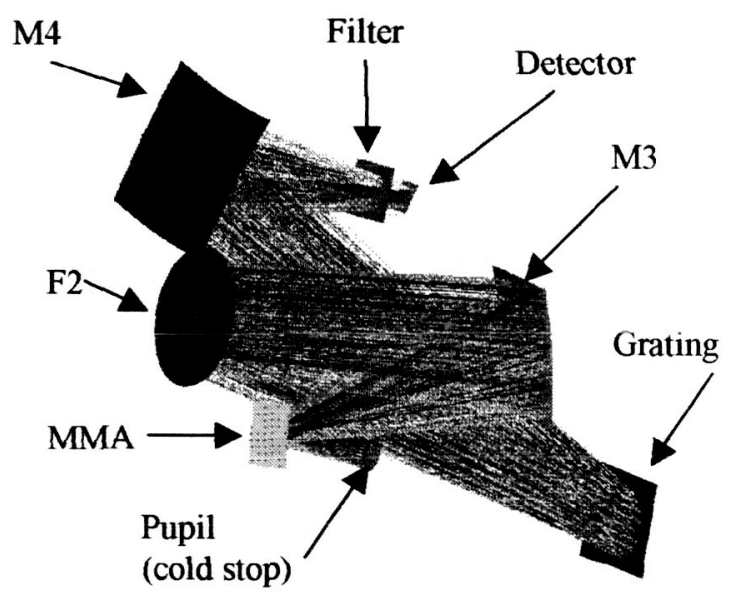

(b)

Figure 1. (a) Side view of stage 1 (focal reducer). Light travels from the telescope focal plane to the MMA. (b) side view of stage 2 (spectrograph). Light travels from the MMA to the detector.

The performance of the two stages is measured independently in a breadboard setup. The performance metrics are the size and morphology of the PSF. Mirror surface errors with different spatial periods cause broadening of the PSF in different ways. We divide surface error into three categories: mid-frequency error, micro-roughness, and figure error. Mid-frequency error causes near angle scatter, micro-roughness causes wide angle scatter, and figure error broadens the core of the PSF beyond the effects of aperture diffraction and the system optical prescription. The IRMOS optics are designed to yield negligible degradation of image quality (unless atmospheric seeing is better than 0.6 arcsec) thus requiring a PSF with full width at half maximum (FWHM) $\leq 2$ pixels, and a goal of $80 \%$ of the energy in a $3 \times 3$ pixel array.

Further motivation for subsystem breadboard alignment and test is to practice and evaluate the alignment procedure intended for the full instrument. The procedure uses alignment datum surfaces and fiducials on the mirror and mechanism substrates. The alignment procedure is easier to troubleshoot and validate when the components are easily accessible and not yet part of a compact, populated optical bench.

We present the IRMOS mechanical design, the optomechanical alignment procedure, results from breadboard alignment and image testing, cryogenic characterization of the instrument bench, population of the bench with mirrors and 
mechanisms, ambient instrument end-to-end image testing without the MMA, and a prediction of image size and slit transmission for the cryogenic instrument.

\section{MECHANICAL DESIGN}

This section gives a brief summary of instrument mechanical design. Winsor et al., Ohl et al., and Hylan et al., present a detailed description of the optical prescription, mirror optomechanical design, and system mechanical design., ${ }^{2,3,6}$

The optical bench and mirror substrates are made of aluminum 6061-T651. This single material choice means the instrument is thermally homogenous and the design is dimensionally predictable in all dimensions. The IRMOS optical design has no plane or axis of symmetry, so the bench holds the optics at compound angles with respect to each other. The off-axis aspheric surfaces and lack of symmetry of the overall design required an opto-mechanical alignment plan founded on the precision fabrication and measurement of datum (or fiducial) features on each component (section 3 ).

Each mirror has an aspect ratio of $\sim 4: 1$. The mirror substrates include semi-kinematic integral flexure mounts. The rear surface and mounting flexures for M1 and M2 are shown in figure 2. During fabrication, the mirrors undergo a stress relief process to minimize figure error change from room temperature to $80 \mathrm{~K}$. The orientation of the plane of the rear surface of each mirror is designed to be approximately perpendicular to the average normal to the front optical surface. This allowed the thickness of the mirror substrate to be approximately constant.

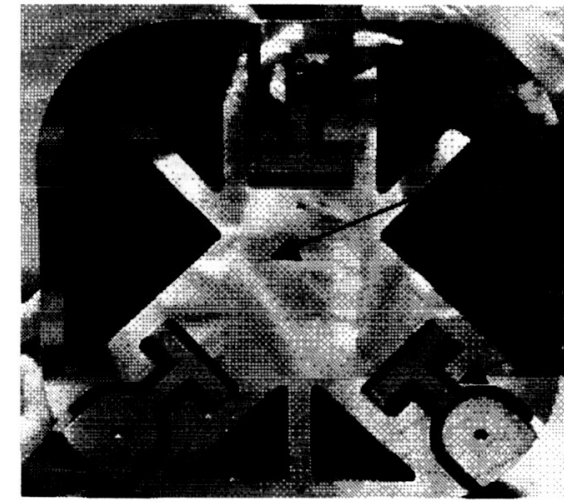

(a)

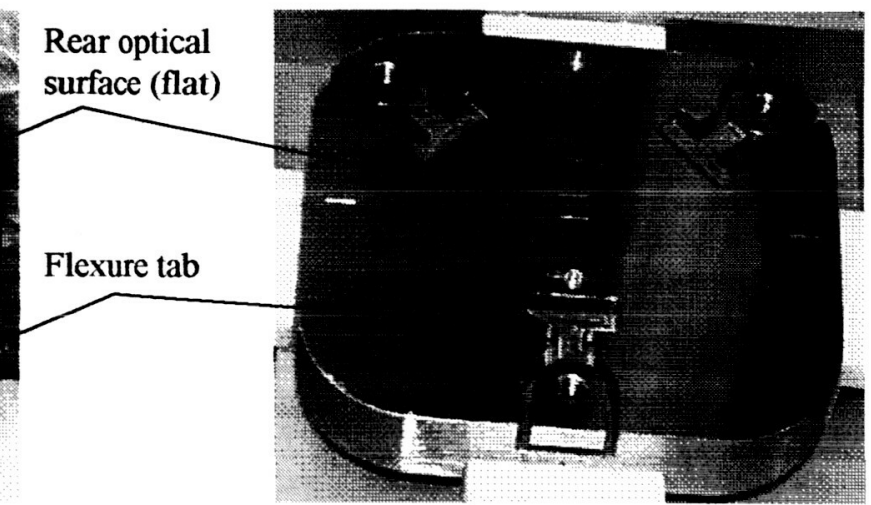

(b)

Figure 2. (a) Rear surface of M2 mirror. Note mounting flexures and flat, diamond machined surface. (b) Rear surface of M1 mirror.

The optical bench is housed in a cylindrical dewar, and consists of two plates ("top" and "bottom") connected by four longerons. ${ }^{6}$ We define an instrument alignment coordinate system (ACS) for assembly purposes. The ACS is oriented such that the $\mathrm{z}$ axis points into the instrument, and the $\mathrm{y}$ axis points toward the instrument dewar window (which is not centered in the top plate). The ACS is defined by several sets of fiducial scribes and datum surfaces on the end plates of the instrument bench, and has its origin at the center of the top plate. An alignment cube is bolted to the bench, and its orientation is measured with respect to the machined fiducial marks on the top plate. The alignment cube serves as an optical rotation reference for orienting objects with respect to the ACS. The scribe fiducials on the bench define the translation origin.

Similarly, each mirror is equipped with a set of optomechanical alignment fiducials that allow alignment to the ACS in six degrees of freedom. Since the powered mirrors are off-axis aspheres, the fiducials are placed on the optic during fabrication in a manner that can be related to the origin of the parent surface or vertex. Each mirror has three sets of alignment fiducials or datum surfaces:

1. The rear surface of the substrate is diamond machined flat to $<0.25 \lambda$ RMS $(\lambda=632.8 \mathrm{~nm})$.

2. Three thin scribe crosshairs are machined onto the rear surface of the substrate (figure 3 ). The fiducials lie along a line that is perpendicular to a line through the center of the mirror aperture and the vertex. 
3. One crosshair fiducial is inscribed on each side of the rectangular substrate (figure 3).

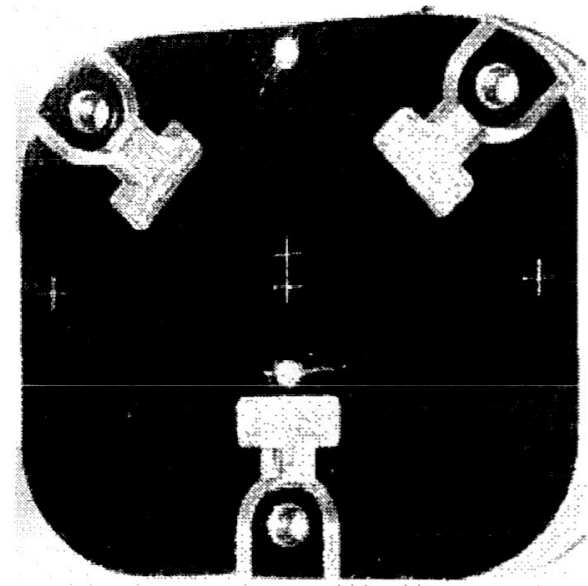

(a)

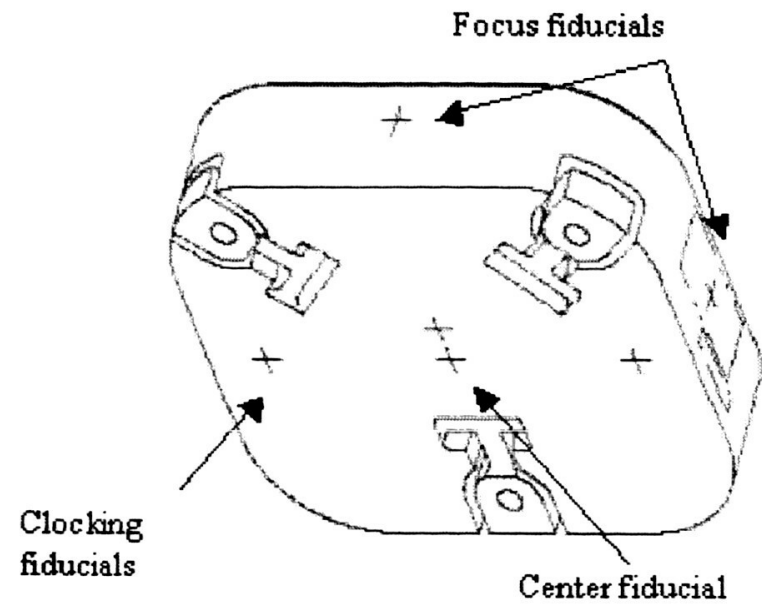

(b)

Figure 3. (a) Photo of rear of M1. (b) Schematic of the rear of a typical mirror substrate. The center fiducial is at the center of the mirror aperture as projected parallel to the optical axis.

\section{SUBSYSTEM ALIGNMENT AND VERIFICATION}

\subsection{Breadboarding}

After component acceptance testing, we assemble stage 1 and 2 independently on a breadboard, in a class 10,000 clean room. Breadboarding allows flexibility and maneuverability in buildup (bench and mechanisms are out of the way), proof of the optical design, and practice using optomechanical alignment techniques.

Since each subsystem is assembled and tested independently, we define a breadboard coordinate system (BCS) with a separate origin for each. For stage 1, we choose datum surfaces on M2, and for stage 2, we choose datums on M4. For both optics, the optically flat rear surface serves as the tip and tilt reference plane, the three clocking fiducials on the rear serve as the clocking reference, the center fiducial serves as the reference for two translational degrees of freedom, and the side "focus" fiducial serves as the third. Several pieces of hardware are included in the breadboard assembly: a $1.2 \times 0.9 \mathrm{~m}$ breadboard made of flat $( \pm 50 \mu \mathrm{m})$ mic- 6 aluminum jig plate, a precision rotary table, ${ }^{*}$ a $1.2 \mathrm{~m} \times 2.4 \mathrm{~m}$ flat $( \pm 0.025 \mathrm{~mm})$ granite table, and tip/tilt plates above and below the rotary table for leveling its axis of rotation and the plane of the breadboard. The gravity vector is perpendicular to the plane of the breadboard and to the rotation plane of the rotary table.

A cathetometer and the rotary table are used in the alignment process for precise translational measurement. The cathetometer consists of an alignment telescope mounted to a large $(800 \mathrm{~mm}$ range) two-axis $x-y$ translation stage. Its axes and telescope base ray are highly orthogonal. The cathetometer is aligned to the BCS in three rotational degrees of freedom, via an alignment cube reference bonded to its side. The telescope points perpendicular to the plane defined by the axes of the cathetometer, and directly along one axis of the BCS. The telescope translates horizontally along the second axis of the BCS, and translates vertically along the third axis of the BCS (parallel to the gravity vector). Theodolites are used to provide precise angular measurements. This optomechanical alignment setup and procedure is similar to that developed for previous NASA/GSFC missions, and is shown schematically by Connelly et al. ${ }^{5,8}$

\footnotetext{
* AA Gage, Sterling Heights, Michigan, tel.: 810-977-9000, http://www.metrologyworld.com/storefronts.html
} 
The optomechanical alignment procedure for each stage involves a successive addition of optics to the breadboard. each mounted on stages to control 6 degrees of freedom. For example, for stage 1, M2 is placed on the breadboard first because it is the largest and heaviest mirror in the instrument $(8 \mathrm{~kg})$. Since $\mathrm{M} 2$ is difficult to move, its location in translation defines the reference point of the BCS. Clocking $\left(R_{Z}\right)$ of $M 2$ is set by the cathetometer and clocking fiducials on the rear of the optic. The optic is clocked until those fiducials lie along a level line with respect to gravity. The rear surface of $M 2$ is aligned to gravity and to the plane of the cathetometer in tip and tilt $\left(R_{X}\right.$ and $\left.R_{y}\right)$ using a theodolite in autocollimation and the cathetometer cube. M1 is the next optical element placed on the breadboard. M1 and F2 are aligned similarly in rotation, and are set in translation using the M2 center clocking fiducials as a reference point. The instrument ray trace model generated with ZEMAX is used to determine the angle and offset of each optic (M1 and F1) with respect to the origin (M2). The error in aligning each component in the BCS is $\pm 0.125 \mathrm{~mm}$ in translations and \pm 5 arcsec in rotations. The errors are dominated by the apparent size of the scribe alignment fiducials, alignment instrument calibration, and operator error.

\subsection{Interferometric analysis}

After optomechanical alignment is completed, we use a laser unequal path interferometer (LUPI) to measure wavefront error. ${ }^{9,+}$ The software package Durango ${ }^{\ddagger}$ is used to control the interferometer and obtain wavefront measurements.

The interferometric alignment verification procedure is as follows : First the LUPI is placed on the breadboard in tip and tilt such that its optical axis points directly along the path of the chief ray from the KPNO telescope at a given field point (with respect to the origin of the stage being tested). Next, the focus position of the telescope is located using a $5 \mu \mathrm{m}$ pinhole aligned in $x, y$, and $z$ using the cathetometer. A spherical lens is attached to the LUPI, and the focus of the lens is translated to the location of the pinhole. The LUPI is locked down and the pinhole is removed. A spherical, reflective tooling ball ( $19 \mathrm{~mm}$ diameter convex mirror) is placed at the approximate focus of the subsystem using the cathetometer (i.e., in place of the MMA for stage 1) such that we obtain an interferogram from the subsystem in double-pass. The tooling ball is moved in small translations until the interference pattern is optimized. ${ }^{10}$ Finally, we compare the measured wavefront to that predicted by ZEMAX ray trace modeling.

The measured and modeled interferograms for stages 1 and 2 are shown below in figure 4. Due to worse than expected figure, mid-frequency, and micro-roughness on the powered optics (sampled twice during the double pass test), we are unable to quantitatively compare the measured and modeled wavefront. The effect of tool marks on M1 and M2 are clearly evident in the measured interferogram (figure 4b). However, careful qualitative inspection (fringe counting) indicates that we have obtained results similar to those expected, to within a factor of two. Potential causes of this difference include small but known system misalignment, incomplete characterization of mirror fiducials on a component level, and inadequacy of the modeled Zernike fit to figure error data. ${ }^{4}$

\section{SUBSYSTEM IMAGE TESTING}

\subsection{Image testing}

Image testing is performed at two wavelengths: $546.1 \mathrm{~nm}$ (although out of band) is chosen for ease of alignment, and $1550 \mathrm{~nm}$ is chosen for in-band performance. A mercury argon pencil lamp is used for the visible line, and a blackbody source is used for the infrared line. The image test provides final verification of alignment (i.e., PSF size and morphology) and provides a measure of scatter caused by small scale surface error on the mirrors. Near angle scattering is worse in the visible than it is in the infrared, so measurements of scattered light at $546.1 \mathrm{~nm}$ provide a conservative estimate of instrument performance in the infrared. Image tests are performed at several field points. The field point array is defined as a $3 \times 3$ array, with the center field point called "field point 5." As you look from the telescope into the

\footnotetext{
* Focus Software Inc., Tucson, Arizona, tel.: 520-733-0130, http://www.zemax.com

${ }^{+}$Buccini Instrument Company, Wilmington, North Carolina, tel.: 910-350-1968

${ }^{\ddagger}$ Diffraction International Inc., Minnetonka, Minnesota, tel.: 952-945-9912, http://www.diffraction.com
} 
instrument, the upper left field point is called "field point 1 ," and the lower left field point is called "field point 3." Field points 7 and 9 are at upper right and lower right, respectively.

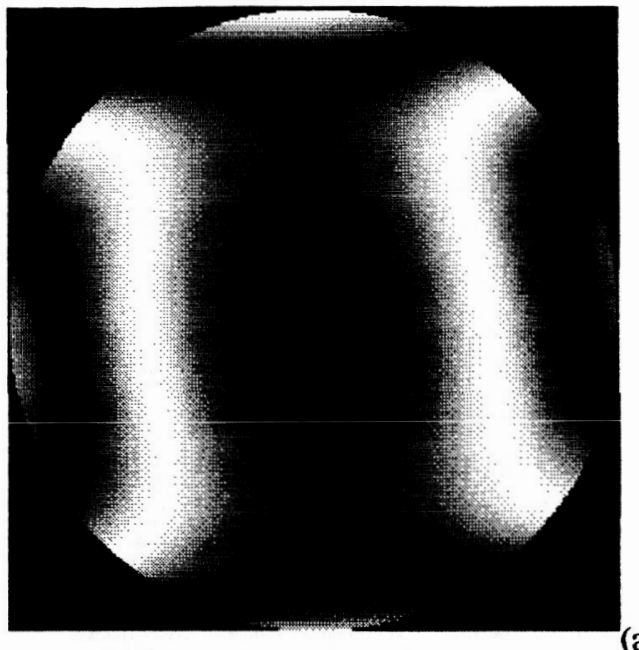

(b)
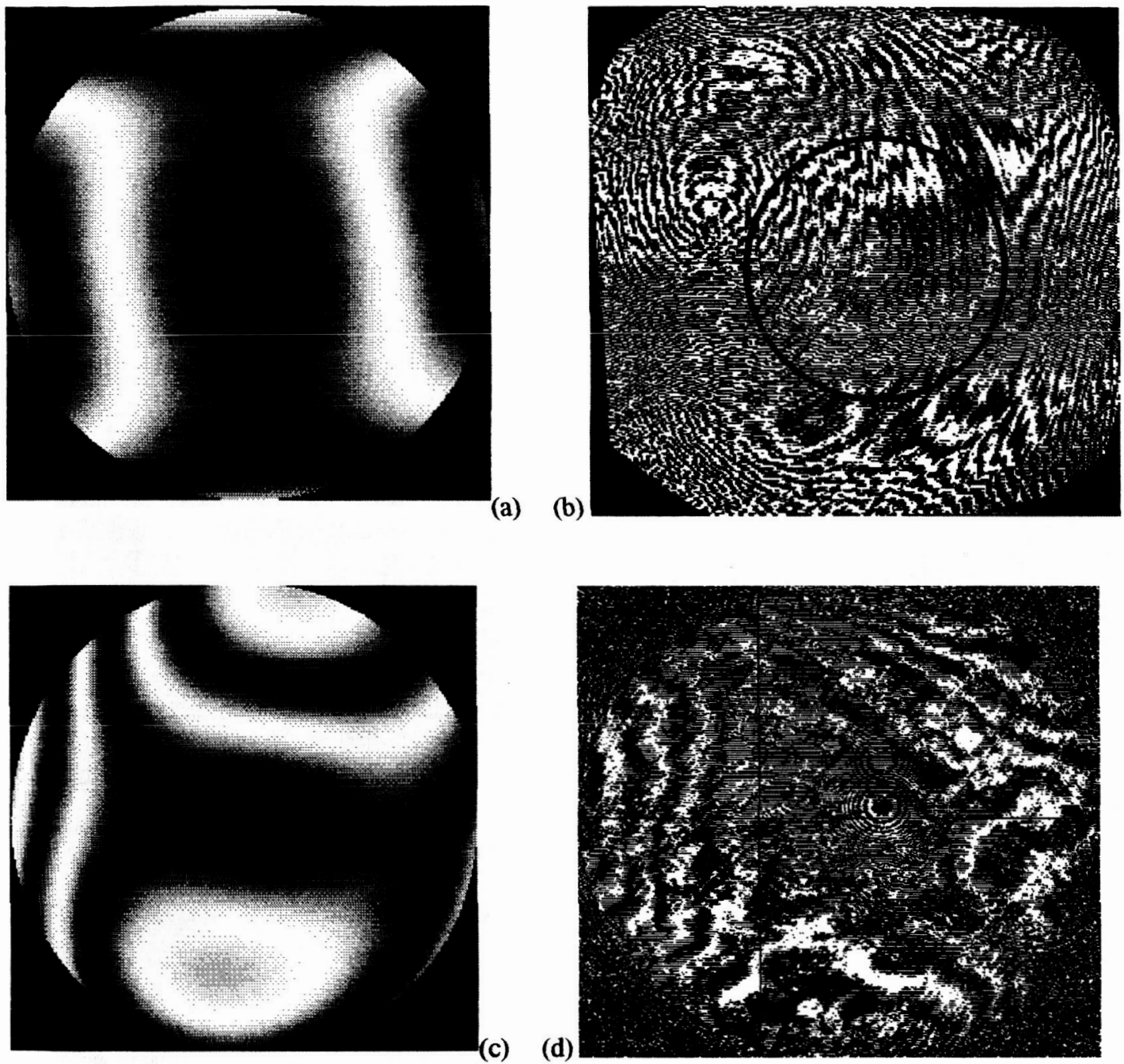

(d)

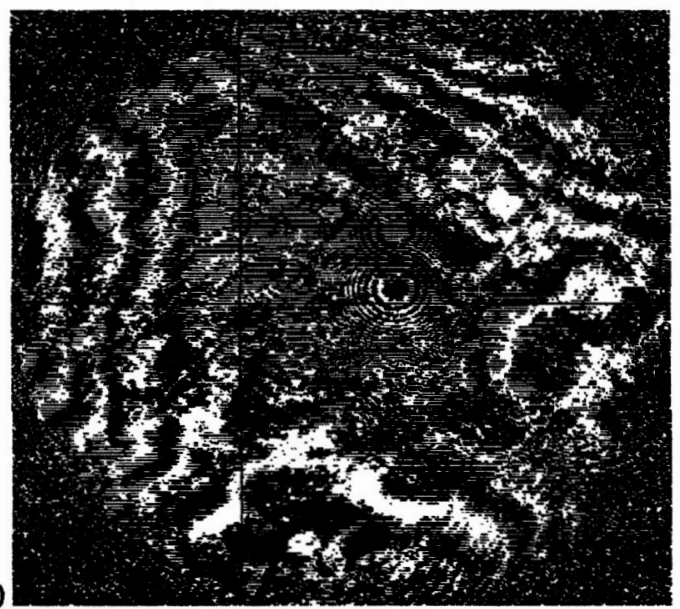

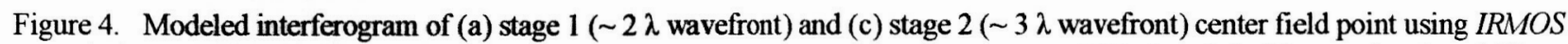
prescription and 36 term Zernike fit to mirror figure error data. Measured interferogram of (b) stage 1 ( $~ 8 \lambda$ wavefront) and (d) stage $2(\sim 6 \lambda$ wavefront). All interferograms at $\lambda=632.8 \mathrm{~nm}$.

The image test procedure has four steps: First, a CCD camera* is placed at the location of the MMA (i.e., the focus of the stage 1 subsystem). The focus of the lens on the front of the LUPI is imaged to rough align the camera. The camera is focused with micron-sized steps using DC motor actuators controlled by custom LabVIEW ${ }^{+}$software. We use commercial image processing software to fit a two-dimensional Gaussian to the image, and the camera is translated until the full width at half maximum of the imaged spot is minimized. ${ }^{\ddagger}$ Next, a $5 \mu \mathrm{m}$ pinhole is placed at the focus of the LUPI. The LUPI is removed and a mercury-argon (HgAr) pencil lamp is positioned behind the $5 \mu \mathrm{m}$ pinhole. The $\mathrm{HgAr}$ pencil lamp provides the spectral line at $546.1 \mathrm{~nm}$. Next, an aperture stop is placed in front of the lamp to stop the beam down to $\mathrm{f} / 15$, to simulate an object incoming from the KPNO telescope. Finally, a $546.1 \mathrm{~nm}$ interference filter is

\footnotetext{
* Apogee Instruments, Auburn, California, tel.: 530-888-0500, http://www.ccd.com

+National Instruments, Austin, Texas, tel: 512-683-0100, http://www.ni.com

${ }^{\ddagger}$ Diffraction Limited, Ottawa, Ontario, tel.: 613-225-2732, http://www.cyanogen.com
} 
attached to the front of the CCD camera. Dark, bias, background, and raw image data are obtained. Prior to final imaging, all exposed surfaces are covered with an opaque material (black plastic or black tape). The room lights are turned off, and all auxiliary light sources are baffled. A black box was constructed and covers the entire breadboard.

\subsection{Measured and modeled results}

Image data is processed using custom routines written in the Interactive Data Language (IDL).* The program applies bias, dark, and background subtractions, locates the image centroid, and generates line profiles, encircled energy curves, and FWHM values. We use ZEMAX to predict RMS spot size. For both subsystems, at all field points, and at both wavelengths, the measured results agree with the modeled results to within the error bars $(30 \mu \mathrm{m} \pm 10 \mu \mathrm{m})$. Together, stage 1 and 2 are designed to image a point source to a spot of $<55 \mu \mathrm{m}$, and all subsystem field points beat this criterion.

We use the Optical Surface Analysis Code (OSAC) to model an encircled energy curve for stage $1 .{ }^{11}$ Stage 1 results are presented in detail by Connelly et al. ${ }^{5}$ We use the Advanced Systems Analysis Program (ASAP) ${ }^{+}$to model an encircled energy curve for stage 2 (OSAC is unable to model non-symmetric systems). The periodic tool marks and surface errors of the optics are approximated as low-order gratings.

\section{BENCH CHARACTERIZATION}

There are two differences between bench integration at GSFC and telescope operation at KPNO. First, integration occurs at ambient temperature and pressure, while operation occurs at $80 \mathrm{~K}$ and vacuum. Second, integration takes place in a horizontal configuration, while operation takes place in a vertical configuration. Prior to integration, we test the effect of each of these differences.

The dewar is thermally cycled three times to test for deviations between the top and bottom plates from ambient to cryogenic temperatures. Mass models are bolted in place of the optics and mechanisms, and optical cubes are attached to the top and bottom plates. Using theodolites and an alignment telescope, we monitor the location of the cubes in six degrees of freedom as the bench cools from $293 \mathrm{~K}$ to $80 \mathrm{~K}$. Results show that the cubes move minimally with respect to each other, less than $0.25 \mathrm{~mm}$ in translation, and less than $10 \mathrm{arcsec}$ in rotation.

We mount two new cubes to the top and bottom plates to test for deviations between the top and bottom plates from vertical to horizontal configuration. In the horizontal configuration, the dewar is held by a large L-bracket via a "slave plate" between the bench and bracket. The rotation offsets between the cubes are measured first without the slave plate (vertical), next with the slave plate (vertical), and finally attached to the slave plate and bench (horizontal). Results show a 20 arcsec sag of the end of the bench not supported by the L-bracket, so a small jack screw is turned to raise this end of the bench until the offset is eliminated.

\section{BENCH POPULATION}

The optical bench is populated sequentially: we begin with stage 1 optics, proceed with stage 2 optics, and finish with the mechanisms, grating wheel, MMA, filter wheel, and focus mechanism. Each optic is aligned via datum surfaces in the manner described above in section 3 . There are two differences during actual bench integration. The origin for all optics is defined via the top plate, and thin aluminum shims are used to adjust tip, tilt, and despace of each optic.

The center of the outer surface of the top plate of the dewar serves as the origin of the alignment coordinate system. Datum surfaces between the dewar and bench are mechanically related such that precise knowledge of the origin is retained, thus datums on the dewar can be used to align IRMOS to the Kitt Peak telescope.

\footnotetext{
- Research Systems Inc., Boulder, Colorado, tel.: 303-786-9900, http://www.rsinc.com

+ Breault Research Organization, Tucson, Arizona, tel.: 520-721-0500, http://www.breault.com
} 
While aligning the subsystems on the breadboard, each optic was mounted with lab stages, and micrometers were used to adjust all degrees of freedom. On the optical bench, we use three point aluminum shims and careful placement to adjust each optic. The optical error budget for alignment is summarized in table 1. Upon initial placement, each optic was $<10$ arcmin from nominal in tip, tilt, and clocking, and $<1 \mathrm{~mm}$ in despace and decenter. All optics equal or better the tolerance values in all degrees of freedom.

\begin{tabular}{|c|c|c|c|c|c|c|}
\hline & Tip (arcsec) & Tilt (arcsec) & Clocking (arcmin) & X (mm) & $Y(\mathrm{~mm})$ & $\mathrm{Z}(\mathrm{mm})$ \\
\hline M1 & $30 / 25$ & $30 / 5$ & $5 / 1$ & $0.13 / 0.03$ & $0.13 / 0.03$ & $0.13 / 0.03$ \\
\hline M2 & $30 / 5$ & $30 / 12$ & $5 / 3$ & $0.13 / 0.03$ & $0.13 / 0.03$ & $0.13 / 0.03$ \\
\hline F1 & $30 / 7$ & $30 / 8$ & n/a & $0.13 / 0.03$ & $0.13 / 0.03$ & $0.13 / 0.05$ \\
\hline M3 & $60 / 20$ & $60 / 20$ & $3 / 1$ & $0.25 / 0.03$ & $0.25 / 0.03$ & $0.25 / 0.03$ \\
\hline M4 & $60 / 30$ & $60 / 5$ & $5 / 2$ & $0.25 / 0.03$ & $0.25 / 0.03$ & $0.25 / 0.03$ \\
\hline F2 & $60 / 30$ & $60 / 30$ & n/a & $0.25 / 0.13$ & $0.25 / 0.05$ & $0.25 / 0.05$ \\
\hline
\end{tabular}

Table 1. Bench integration results: alignment tolerances / as built condition.

Interferometric testing is again used for alignment verification. Our goal is to verify alignment, and to relate the plane of the MMA (plane of best focus of stage 1) to the focal plane of the KPNO telescope. A reflective tooling ball is set at the plane of the MMA for each field point, and the focus position of the LUPI (at the entrance position) is allowed to float until an optimal interferogram is achieved. The planes are at their nominal angle with respect to each other, but are further apart than designed by $2.5 \mathrm{~mm}$. To further verify stage 1 integration, image testing is done at three field points using a CCD camera at the focus of the focal reducer stage. Results are shown in figure 5, where 1-dimensional PSF cuts are plotted in the $\mathrm{x}$ and $\mathrm{y}$ directions $(546.1 \mathrm{~nm})$. We fit a Gaussian to the data in each direction, and calculate the FWHM. For each of the field points tested, and in the $x$ and $y$ directions, the FWHM is between 25 and $40 \mu \mathrm{m}$. Stage 2 optics are aligned, and a high quality flat mirror (MMA surrogate) is set in place of the MMA. Interferometric measurements verify that the system is well aligned.
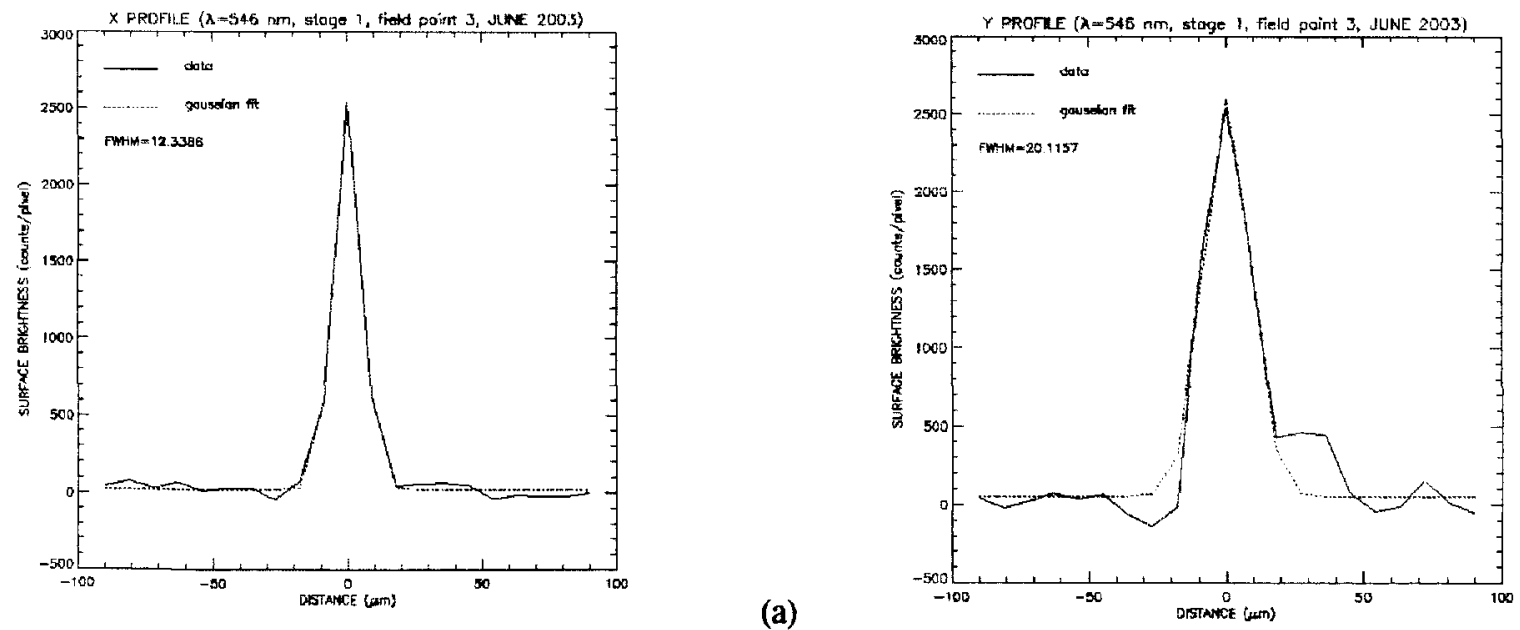

(a) 

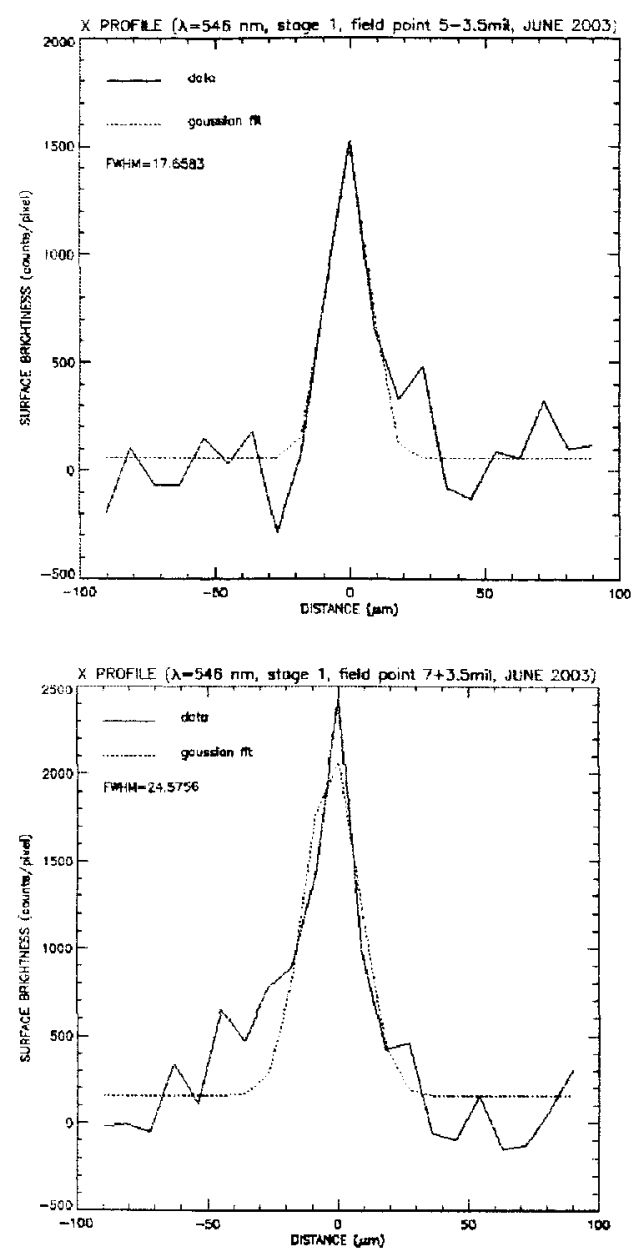

(b)
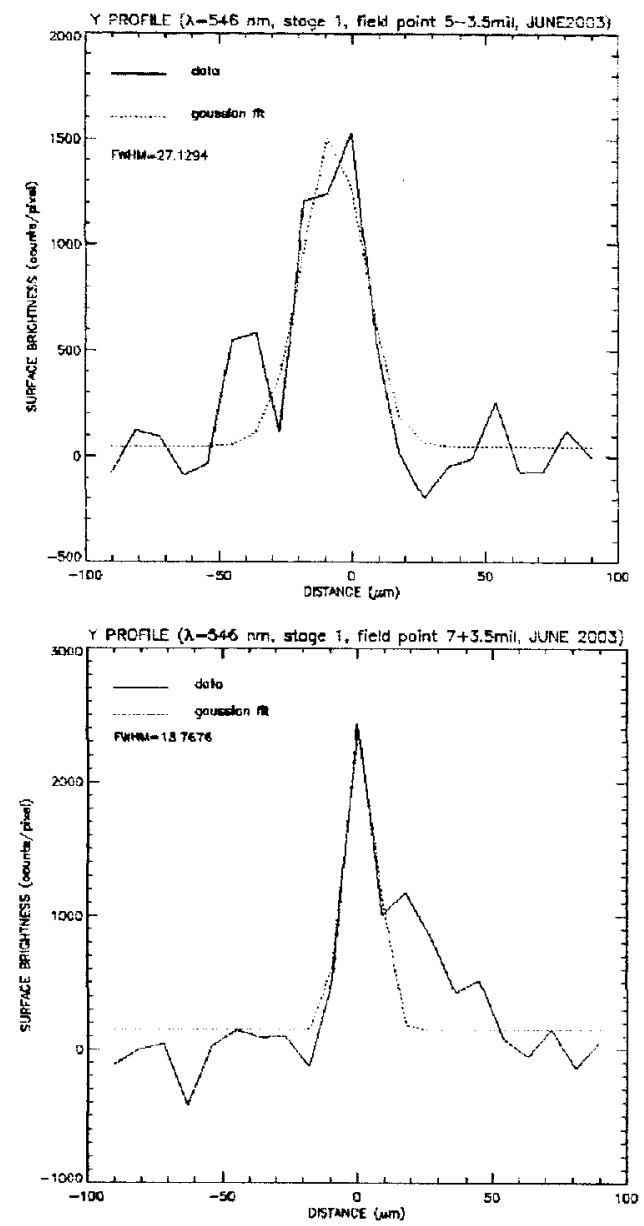

(c)

Figure 5. PSF cuts in the $x$ and $y$ direction for stage 1 at (a) field point 3 , (b) field point 5 , and (c) field point 7 .

\section{INSTRUMENT IMAGE TESTING}

The final verification step is end-to-end image testing. Image tests are run as successive optics and mechanisms are added to the bench. IRMOS is designed for optimal performance at 9 field points, and the points are defined at the KPNO telescope focal plane in a $3 \times 3$ array. We test the center field point during all image tests, and the four corner field points during select image tests. Image tests are conducted at $546.1 \mathrm{~nm}$ (to aid setup, and provide a worst case scenario) and $1550 \mathrm{~nm}$ (in-band) with a backlit $25 \mu \mathrm{m}$ pinhole.

The first end-to-end image test includes stage 1, stage 2, the grating wheel mechanism, and the MMA surrogate. A 1dimensional cut is taken in the $x$ and $y$ directions, and a Gaussian is fit and yields a FWHM of $27 \mu \mathrm{m}$ in the $\mathrm{x}$ direction and $37 \mu \mathrm{m}$ in the y direction $(546.1 \mathrm{~nm})$. Results are shown in figures $6 \mathrm{a}$ and $6 \mathrm{~b}$, with the $\mathrm{x}$-axis plotted in detector pixels (18 $\mu \mathrm{m}$ per pixel). For the infrared image test, a 2-dimensional Gaussian is fit and yields a FWHM of 3.4 detector pixels (figures $6 \mathrm{c}$ and $6 \mathrm{~d}$ ). When "de-RSSed" with the $40 \mu \mathrm{m}$ pixel size of the infrared detector, a real FWHM of 2.6 pixels is obtained. The next image test will include the engineering grade MMA, and the following test will add the focus mechanism with a MUX detector. Further testing includes adding the engineering grade detector, adding the science grade MMA, adding the science grade detector, and finally adding the filter wheel. Once these image tests are complete, the bench is integrated with the dewar. For final image testing, we include sources in the $\mathrm{H}, \mathrm{J}$, and $\mathrm{K}$ bands, and test the center field point and four corner field points at a temperature of $80 \mathrm{~K}$. Thus, the final image test includes 
in-band image testing of stage 1 , stage 2 , the science grade MMA, the grating wheel mechanism, the filter wheel mechanism, the focus mechanism, and the science grade detector, all at a temperature of $80 \mathrm{~K}$.

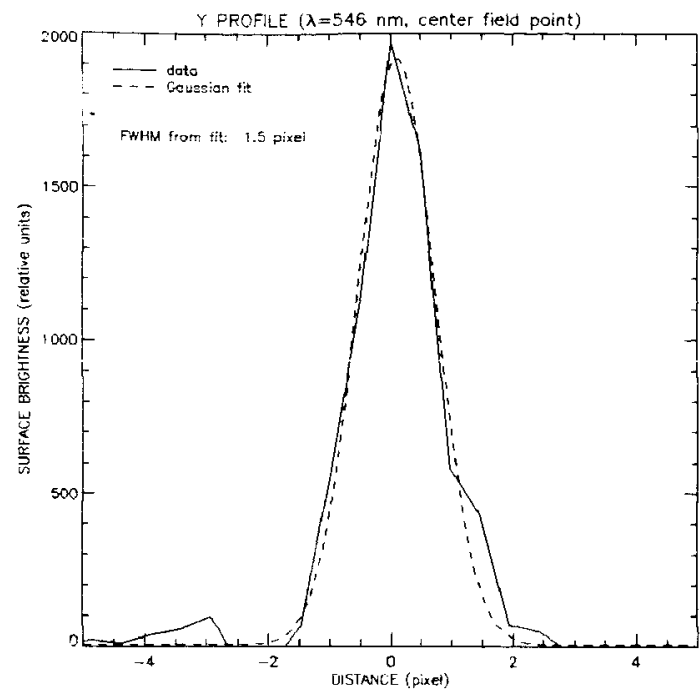

(a)
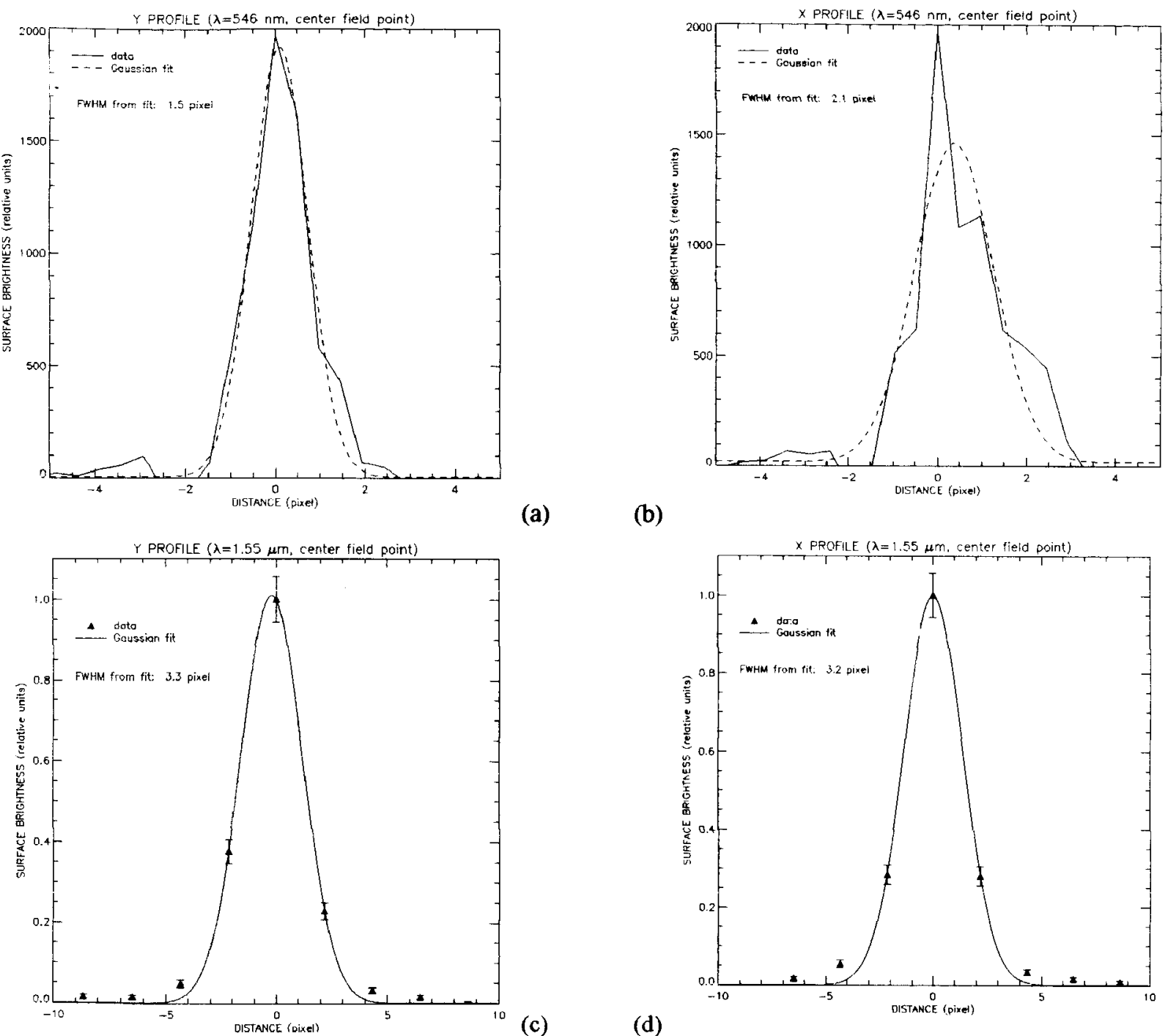

(b)

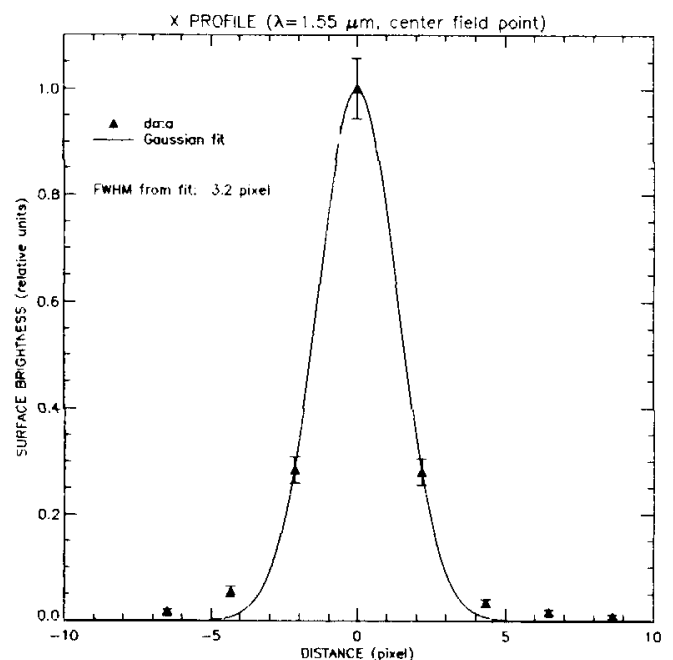

(c) $\quad(d)$

Figure 7. PSF cuts for stage $1+$ stage $2+$ DMD surrogate at field point 5 at $546.1 \mathrm{~nm}$ (a) $y$ and (b) $x$ direction, and at $1550 \mathrm{~nm}$ (c) $y$ and (d) $x$ direction. The $y$-axis in plots (c) and (d) is normalized, and the error bars give a sense of the signal to noise ratio.

\section{CONCLUSIONS}

We test the IRMOS subsystems on a breadboard and verify that they perform as expected, and that the opto-mechanical alignment method works. The subsystems are integrated with the instrument bench, and a rigorous program of optical testing shows that $I R M O S$ performs as well as expected, as ambient testing demonstrated spot sizes of less than $55 \mu \mathrm{m}$ at every field point both at visible and infrared wavelengths. The MMA provides a real-time programmable slit mask, and should not significantly degrade image quality. Following successful dewar integration and cryogenic image testing, IRMOS should be delivered to KPNO in October of 2003 for integration with the $4 \mathrm{~m}$ and $2.1 \mathrm{~m}$ telescopes. 


\section{ACKNOWLEDGEMENTS}

We thank Joseph C. McMann, Thomas J. French, Kevin W. Redman, and Louis R. Worrel Jr. of the Management Technology team for advice, alignment assistance, and fabrication work, and Rene A. Boucarut of GSFC for equipment. This work is funded by the James Webb Space Telescope project and the Center Director's Discretionary Fund at NASA/Goddard Space Flight Center, and by the Director's Discretionary Research Fund at the Space Telescope Science Institute.

\section{REFERENCES}

1. J. W. MacKenty, M. A. Greenhouse, R. F. Green, L. M. Sparr, R. G. Ohl, and R. S. Winsor, "IRMOS: An infrared multi-object spectrometer using a MEMS micro-mirror array," Proc SPIE 4841, pp. 953-961, 2002.

2. R. S. Winsor, J. W. MacKenty, M. Stiavelli, M. A. Greenhouse, J. E. Mentzell, R. G. Ohl, and R. F. Green, "Optical design for an infrared multi-object spectrometer," Proc. SPIE 4092, pp. 102-108, 2000.

3. R. G. Ohl, W. Preuss, A. Sohn, S. Conkey, K. Garrard, J. G. Hagopian, J. M. Howard, J. E. Hylan, S. M. Irish, J. E. Mentzell, M. Schroeder, L. M. Sparr, R. S. Winsor, S. W. Zewari, M. A. Greenhouse, and J. W. MacKenty, "Design and fabrication of diamond machined, aspheric mirrors for ground-based, near-IR astronomy," Proc. SPIE 4841, pp. 677-688, 2003.

4. V. J. Chambers, R. G. Mink, R. G. Ohl, J. A. Connelly, J. E. Mentzell, S. M. Arnold, M. A. Greenhouse, R. S. Winsor, and J. W. MacKenty, "Optical testing of diamond machined, aspheric mirrors for ground-based, nearIR astronomy," Proc SPIE 4841, pp. 689-701, 2003.

5. J. A. Connelly, R. G. Ohl, T. T. Saha, T. Hadjimichael, J. E. Mentzell, R. G. Mink, J. E. Hylan, L. M. Sparr, V. J. Chambers, J. G. Hagopian, M. A. Greenhouse, R. S. Winsor, and J. W. MacKenty, "Imaging performance and modeling of the Infrared Multi-Object Spectrometer focal reducer," Proc SPIE 4841, pp. 702_-714, 2003.

6. J. E. Hylan, L. M. Sparr, R. G. Ohl, J. E. Mentzell, M. A. Greenhouse, and J. W. MacKenty, "Lessons learned from the design, fabrication, integration, and test of a cryogenic, IR spectrometer for ground based astronomy," Proc SPIE 5172, 2003.

7. R. W. Toland, R. G. Ohl, M. P. Barthelmy, S. W. Zewari, M. A. Greenhouse, and J. W. MacKenty, "Effects of forged stock and pure aluminum coating on cryogenic performance of heat treated aluminum mirrors," Proc SPIE 5172, 2003.

8. J. G. Hagopian, P. A. Hayes, J. A. Crooke, and J. J. Lyons, "Optomechanical alignment of the Composite Infrared Spectrometer (CIRS) for the Cassini mission to Saturn," Proc. SPIE 2814, pp. 46-58, 1996.

$9 . \quad$ J. B. Houston, C. J. Buccini, and P. V. O'Neill, "A laser unequal path interferometer for the optical shop," Applied Optics 6, pp. 1237-1242, 1967.

10. R. H. Barkhouser and R. G. Ohl, "Interferometric alignment and figure testing of large $(0.5 \mathrm{~m})$ off-axis parabolic mirrors in a challenging cleanroom environment," Proc. SPIE 3782, pp. 601-614, 1999.

11. R. J. Noll, P. Glenn, and J. F. Osantowski, "Optical surface analysis code (OSAC)," Proc. SPIE 62, pp. 78-82, 1983. 